\title{
Caregiver's Education Level and Child's Dental Caries in African Americans: A Path Analytic Study
}

\author{
Masahiro Heima ${ }^{a}$ Wonik Lee ${ }^{a}$ Peter Milgrom ${ }^{b}$ Suchita Nelson ${ }^{a}$

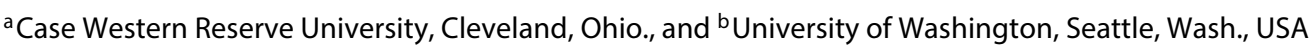

\author{
Key Words \\ Dental caries · Parents · Caregivers · Child · Educational \\ status · African American · Logistic models
}

\begin{abstract}
The objective of this study was to investigate the influence of caregiver education level on children's dental caries mediated by both caregiver and child oral health behaviors. Participants were 423 low-income African American kindergarteners and their caregivers who were part of a school-based randomized clinical trial. Path analysis tested the hypothesis that caregiver education level affected untreated dental caries and cumulative overall caries experience (decayed or filled teeth) through the mediating influence of frequency of dental visits, use of routine care, and frequency of toothbrushing for both the caregiver and the child. The results supported the hypothesis: caregivers who completed high school were 1.76 times more likely to visit dentists compared with those who did not complete high school $\left(\mathrm{e}^{0.56}=1.76\right.$, $95 \% \mathrm{Cl}: 1.03-2.99)$, which in turn was associated with 5.78 times greater odds of dental visits among their children $\left(e^{1.76}=5.78,95 \%\right.$ Cl: 3.53-9.48). Children's dental visits, subsequently, were associated with $26 \%$ fewer untreated decayed teeth compared with children without dental visits $\left(\mathrm{e}^{-0.31}=0.74,95 \% \mathrm{Cl}: 0.60-0.91\right)$. However, this path was not present in the model with overall caries experience. Additionally, caregiver education level was directly associated with $34 \%$ less untreated decayed teeth $\left(e^{-0.42}=0.66,95 \% \mathrm{Cl}\right.$ :
\end{abstract}

$0.54-0.79)$ and $28 \%$ less decayed or filled teeth $\left(e^{-0.32}=0.72\right.$, $95 \% \mathrm{Cl}: 0.60-0.88)$ among the children. This study overcomes important conceptual and analytic limitations in the existing literature. The findings confirm the role of caregiver education in child dental caries and indicate that caregiver's behavioral factors are important mediators of child oral health.

(c) 2015 S. Karger AG, Basel

\section{Introduction}

Among the socioeconomic factors, parental education level is clearly important in influencing the development of dental caries in children, but the way in which this happens is not well understood [Hooley et al., 2012]. In medicine, a patient's education level is the best predictor of health conditions and health care behavior in adults compared with other socioeconomic indicators such as income and employment [Laaksonen et al., 2005; Liu et al., 2011; Winkleby et al., 1990]. Similarly, in dentistry, caregiver education levels have been shown to be associated with children's dental visits, tooth brushing frequency, and dental caries prevalence [Camargo et al., 2012; Edelstein, 2002; Rajab et al., 2002].

Path analysis and structural equation modeling (SEM) have been suggested as techniques for probing the influ-

Trial registration: www.clinicaltrials.gov: NCT01901250.

\section{KARGER 125}

(c) 2015 S. Karger AG, Base

0008-6568/15/0492-0177\$39.50/0

E-Mail karger@karger.com

www.karger.com/cre
Masahiro Heima

Department of Pediatric Dentistry, School of Dental Medicine

Case Western Reserve University

10900 Euclid Avenue, Cleveland, OH 44106-4905 (USA)

E-Mail masahiro.heima@ case.edu 
ence of caregiver education level on oral health in children, including mediators such as oral health behavior [Albert and Nelson, 2011; Newton and Bower, 2005]. However, there are conceptual limitations in previous SEM studies. First, although dental problems in primary teeth lead to future dental caries in permanent teeth [al-Shalan et al., 1997; Dulgergil and Colak, 2012], most path model studies have used children's carious permanent teeth instead of carious primary teeth. Second, most path model studies tested children's behavior as a mediator for their oral health outcomes, but they did not consider the caregiver's behavior, even though parental behavior impacts child oral health behavior [Hooley et al., 2012]. Previous research findings suggest that the parent's oral health behavior, such as dental visits and tooth brushing, has a positive influence on their children's oral health behavior [Camargo et al., 2012; Grembowski et al., 2008; Rajab et al., 2002].

The objective of this study was to investigate a hypothesized path model with caregiver education level as exposure, caregiver and children oral health behavior as mediators, and child dental caries as outcome.

\section{Materials and Methods}

\section{Study Design}

This secondary analysis utilized a cross-sectional design using data collected in a longitudinal study.

\section{Study Sample}

The data was collected as part of a randomized clinical trial conducted at all five elementary schools in an urban school district in the United States. The purpose of the school-based cluster randomized trial was to investigate the effectiveness of xylitol versus placebo gummy bears to prevent dental caries in the primary and permanent teeth of kindergarten children [Lee et al., 2014]. Approximately $96 \%$ of the eligible children received the federal free/reduced cost school lunch program, which is available if the household's gross income is not greater than $130 \%$ of the Federal Poverty Guidelines. The children are predominantly African American (94\%). A total of 562 kindergarteners (aged 5-6 years) were recruited for the original study in September of 2007, 2008, and 2009. Four hundred and forty-one child-caregiver dyads participated in the dental examinations (child) and the questionnaire (caregiver). The sample is representative of all the eligible children and caregivers regarding race (African-American), of those living in an urban school district. Since the study enrolled only 18 children of other ethnicity or race, the present secondary analysis was restricted to the 423 African Americans in the sample. The Institutional Review Board of University Hospitals Case Medical Center approved the study and all caregivers provided written informed consent.

\section{Theoretical Model}

The study is based on the modeling theory that posits people learn their behavior from one another through observation [Bandura, 1969]. We investigated the influence of caregiver educa- tion level on children's decayed primary teeth using a hypothesized causal model with four stages: (1) caregiver education level as antecedent, (2) caregiver's oral health behaviors as a mediator, (3) children's oral health behaviors as a mediator, and (4) the children's dental caries (fig. 1).

\section{Measures}

A caregiver questionnaire, which was written in English at the 6th grade reading level, was completed by the primary caregiver. The questions were adapted from the National Health and Nutrition Examination Survey III (NHANES III) and the Detroit Dental Health Project [Ismail et al., 2008]. When the primary caregivers were enrolled in the original study, they received the questionnaire from a research assistant at their child's school and the questionnaires were completed at home. The primary caregivers were primarily biological mothers. The questionnaire is available from co-author, Nelson.

\section{Caregiver Education Level}

The highest level of education completed was asked in an openended question: 'what is the highest level of education that you completed?' For this study, we dichotomized these answers to whether the caregiver completed high school (yes/no).

\section{Caregiver's and Child's Oral Health Behavior}

Six questions captured caregiver and child oral health behaviors: whether the caregiver or child visited the dentist at least once a year (vs. less than once per year), whether the caregiver or child used routine dental care (vs. problem or symptom driven use), and whether the caregiver or child brushed her/his teeth at least twice a day (yes/no).

\section{Dental Examination}

Dental examinations were conducted in September of each year at school by four to five annually calibrated dentists using a portable dental chair with dental lights and compressed air. Dental caries was assessed in accordance with the International Caries Detection and Assessment System (ICDAS) [Pitts, 2004]. The inter- and intra-rater reliability for the examiners was good to excellent with a kappa $>0.70$ [Nelson et al., 2011]. The number of decayed primary teeth $(\mathrm{dt})$ representing untreated dental caries, and the number of decayed or filled primary teeth $(\mathrm{dft})$ representing cumulative dental caries experience was measured. A decayed tooth was defined as a tooth with ICDAS severity score of 1 (first visual change in enamel) to 6 (extensive cavity) for both measures. The total number of primary teeth present was counted to be included as a covariate.

\section{Statistical Analysis}

Bivariate analyses were conducted to describe the sample characteristics (demographics, oral health behaviors, dental caries) between caregivers who completed high school education and those who did not.

To test the hypothesized causal model in four stages (fig. 1), path analysis was conducted with the following assumptions: bernoulli/logistic regression models for binary outcomes (both caregiver's and child's dental visit once a year, routine dental care visits, and brushing teeth behavior) and a negative binomial distribution/log linear models for untreated decayed teeth (dt) and cumulative dental caries experience $(\mathrm{dft})$. Model coefficients were estimated by maximum likelihood with Monte Carlo simulation for numerical integration, and the corresponding estimated odds 


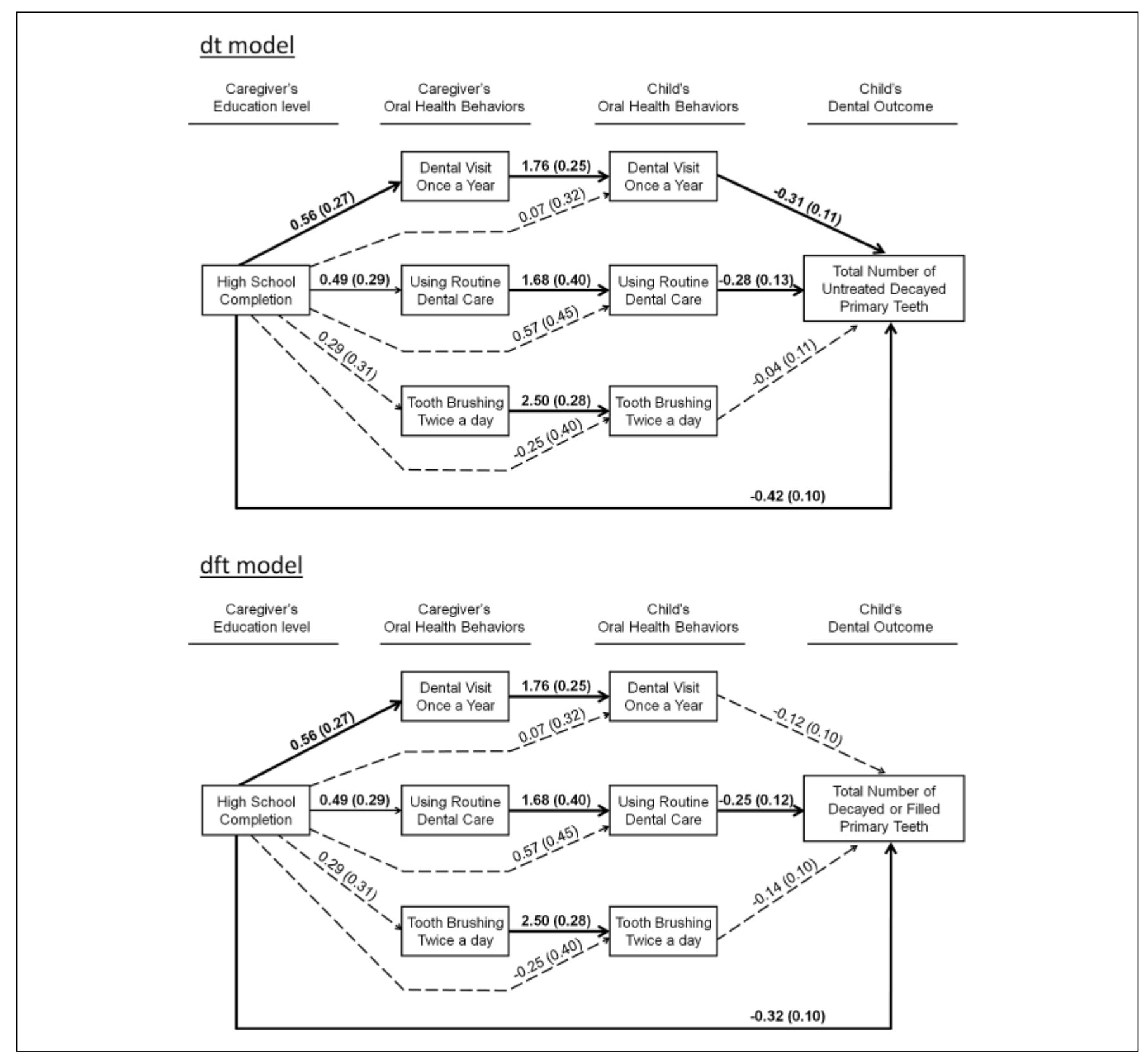

Fig. 1. Path models, $\mathrm{dt}$ model and $\mathrm{dft}$ model, show pathways of the influence of caregiver education level on children's oral health mediated by caregiver's and children's oral health behavior $(n=423)$. It is configured in four stages: (1) caregiver education level as exposure, (2) caregiver's oral health self-care behaviors as mediator, (3) children's oral health behaviors as mediator, and (4) children's oral health outcome, the total number of decayed primary teeth in

ratio (for the logistic regression model) and incidence rate ratio (for the negative binomial model) were obtained as the log of the estimated coefficient. The goodness-of-fit of the path model was assessed by Akaike Information Criteria (AIC) and Bayesian Information Criteria (BIC). There was less than $6.8 \%$ missing data

Influence of Caregiver Education Level on Child's Caries the $\mathrm{dt}$ model and the total of number of decayed or filled primary teeth in the $\mathrm{dft}$ model. Thick continuous line arrows denote the presence of a statistically significant $(\mathrm{p}<0.05)$ relationship. Thin continuous line arrows denote the presence of a trend in significance at $\mathrm{p}<0.10$. Arrows with dotted lines denote the nonsignificant relationships. Estimated $\beta$-coefficients with the standard errors for the estimates (in parentheses) are displayed.

for any variable. To use all available data and maintain the maximum sample size, the multiple imputation of missing data was performed using Bayesian analysis [Sterne et al., 2009]. All analyses of this study were done by using Mplus Version 6 (Muthén and Muthén, Los Angeles, Calif., USA). 
Table 1. Comparisons of demographics, caregiver's oral health behavior, children's oral health behavior, and children's dental outcomes between caregivers with and without high school completion

\begin{tabular}{|c|c|c|c|}
\hline \multirow[t]{2}{*}{ Variables } & \multicolumn{3}{|c|}{ Caregiver educational level } \\
\hline & $\begin{array}{l}\text { complete high school } \\
(\mathrm{n}=327)\end{array}$ & $\begin{array}{l}\text { incomplete high school } \\
(\mathrm{n}=68)\end{array}$ & $\mathrm{p}$ value \\
\hline \multicolumn{4}{|l|}{ Demographics } \\
\hline Caregiver's age & $33.1 \pm 10.3$ & $31.1 \pm 12.6$ & 0.17 \\
\hline \multicolumn{4}{|l|}{ Child's gender, \% } \\
\hline Female & $171(52.3)$ & $34(50)$ & 0.73 \\
\hline Male & $156(47.7)$ & $34(50)$ & \\
\hline \multicolumn{4}{|l|}{ Caregiver's oral health behaviors } \\
\hline \multicolumn{4}{|l|}{ Dental visit once a year, $\%$} \\
\hline Yes & $225(69.7)$ & $39(57.4)$ & $0.05^{*}$ \\
\hline No & $98(30.3)$ & $29(42.6)$ & \\
\hline \multicolumn{4}{|l|}{ Routine dental care, $\%$} \\
\hline Yes & $240(76.2)$ & $43(67.2)$ & 0.13 \\
\hline No & $75(23.8)$ & $21(32.8)$ & \\
\hline \multicolumn{4}{|l|}{ Tooth brushing twice a day, $\%$} \\
\hline Yes & $258(79.9)$ & $51(75.0)$ & 0.36 \\
\hline No & $65(20.1)$ & $17(25.0)$ & \\
\hline \multicolumn{4}{|l|}{ Children's oral health behaviors } \\
\hline \multicolumn{4}{|l|}{ Dental visit once a year, \% } \\
\hline Yes & $241(76.3)$ & $46(69.7)$ & 0.26 \\
\hline No & $75(23.7)$ & $20(30.3)$ & \\
\hline \multicolumn{4}{|l|}{ Routine dental care, $\%$} \\
\hline Yes & $287(92.9)$ & $54(87.1)$ & 0.13 \\
\hline No & $22(7.1)$ & $8(12.9)$ & \\
\hline \multicolumn{4}{|l|}{ Tooth brushing twice a day, $\%$} \\
\hline Yes & $250(77.4)$ & $52(77.6)$ & 0.97 \\
\hline No & $73(22.6)$ & $15(22.4)$ & \\
\hline \multicolumn{4}{|l|}{ Children's dental outcomes } \\
\hline Total number of primary teeth & $18.47 \pm 2.01$ & $18.58 \pm 1.97$ & 0.68 \\
\hline Total number of decayed primary & & & \\
\hline teeth $(\mathrm{dt})$ & $2.77 \pm 3.24$ & $4.81 \pm 3.78$ & $<0.001^{*}$ \\
\hline Total number of decayed or filled & & & \\
\hline primary teeth $(\mathrm{dft})$ & $3.57 \pm 3.68$ & $5.35 \pm 4.16$ & $<0.001^{*}$ \\
\hline
\end{tabular}

\section{Results}

\section{Sample Characteristics}

The sample consisted of 423 children (the mean \pm SD of age was $5.2 \pm 0.4$ at enrollment in September of each year) with $51 \%$ female and $49 \%$ male. The mean age of caregivers was $32.7 \pm 10.7$ years and $86 \%$ of the caregivers were biological mothers, $6 \%$ were biological fathers, and $8 \%$ were other relatives such as adopted or foster parents, or grandparents. Among the caregivers, 327 (77\%) re- ported that they completed high school, $68(16 \%)$ reported that they did not complete high school, and 28 (7\%) did not report.

\section{Bivariate Analysis}

Table 1 gives the results of bivariate analyses of caregiver and child characteristics and health behaviors for caregivers who did or did not complete high school. Caregivers who completed high school were more likely to visit a dentist compared with those who did not complete high 
school. A higher mean number of untreated decayed primary teeth $(\mathrm{dt})$, and cumulative oral health status (number of decayed or filled primary teeth, $\mathrm{dft}$ ) were found in children whose caregivers had not completed high school. There was no difference between the two groups for other caregiver or any child oral health behaviors.

\section{Path Analysis}

The results of path analyses support the hypothesis that caregiver education level affects child dental caries, mediated by oral health behaviors of caregivers and their children (fig. 1). The model with the number of untreated decayed teeth (dt) as a final outcome provided a slightly better model fit (lower scores on AIC and BIC) compared with the model with the number of decayed or filled teeth (dft) as a final outcome.

Effects of Caregiver Education Level on Oral Health Behavior

Caregivers who completed high school were 1.76 times more likely to visit dentists yearly (OR: $\mathrm{e}^{0.56}=1.76,95 \%$ CI: 1.03-2.99) compared with those who did not complete high school. Caregivers' dental visits were, in turn, associated with 5.78 times greater odds (OR: $\mathrm{e}^{1.76}=5.78,95 \% \mathrm{CI}$ : 3.53-9.48) of dental visits for their children. In addition, caregivers who used routine dental care for themselves (vs. problem or symptom driven use) were more likely to bring their children for routine dental care (OR: $\mathrm{e}^{1.68}=$ 5.37, 95\% CI: 2.47-11.67). Similarly, the caregivers who brushed their teeth at least twice a day had 12.2 times greater odds of having children who brushed their teeth at least twice a day (OR: $\mathrm{e}^{2.50}=12.2,95 \%$ CI: 6.97-21.15).

Effects of Oral Health Behavior on Dental Caries

In the df model, children with dental visit once a year (vs. less than yearly) had an estimated 26\% lower mean number of untreated decayed primary teeth compared with those without dental visits (IRR: $\mathrm{e}^{-0.31}=0.74,95 \% \mathrm{CI}$ : $0.60-0.91$ ) and children using routine dental care (vs. problem or symptom driven use) had an estimated $24 \%$ lower mean number of untreated decayed primary teeth compared with those not using routine dental care (IRR: $\mathrm{e}^{-0.28}=0.76$, 95\% CI: 0.59-0.98) controlling for other behavior and caregiver education level. In the dft model, however, routine use of dental care (vs. problem or symptom driven use) was significantly associated with $\mathrm{dft}$, that is, children who use routine dental care had an estimated $22 \%$ lower mean number of decayed or filled teeth compared with those not using routine care (IRR: $\mathrm{e}^{-0.25}=0.78$, 95\% CI: 0.62-0.98). Dental visit once a year was not associ-

Influence of Caregiver Education Level on Child's Caries ated with a difference in the cumulative overall caries experience. Brushing behavior did not show significant associations with dental caries in both the $\mathrm{dt}$ and $\mathrm{dft}$ models.

Direct Effects of Caregiver Education Level on

Children's Dental Caries

Children with caregivers who completed high school had an estimated $34 \%$ lower mean number of dt (IRR: $\mathrm{e}^{-0.42}=0.66,95 \%$ CI: $\left.0.54-0.79\right)$ and $28 \%$ lower mean number of dft (IRR: $\mathrm{e}^{-0.32}=0.72,95 \%$ CI: $\left.0.60-0.88\right)$ compared with children whose caregivers did not complete high school after controlling for all oral health behaviors in the model.

\section{Discussion}

This study highlights two significant pathways through which caregiver education is related to children's dental caries. In the first pathway, lower caregiver education (failure to complete high school) is related to increased untreated caries (dt) through the mediating influence of caregiver and child dental visit once a year in the untreated caries (dt) model. In the second pathway, caregiver and child routine dental care visit behavior mediates the relationship between caregiver education and dental caries outcome (both $\mathrm{dt}$ and $\mathrm{dft}$ ). In the caries experience (dft) model, the only significant path was routine (vs. symptomatic) care mediating the effect of caregiver education on the outcome measure.

While we cannot directly compare our results to prior studies because they did not utilize path-modeling approaches, our findings generally support the results of previous research regarding parental dental visit behavior influencing children's dental visit behavior [Camargo et al., 2012; Grembowski et al., 2008]. Our findings highlight the importance of caregiver education level enhancing caregiver's dental visit behavior and ultimately caries treatment.

Our subjects were from low-income African American families that have poor dental access. Although treatment-related dental visit behavior does not help to reduce the number of children's dental caries, severe dental infection can be prevented, especially for low-income populations with poor dental access.

\section{The Effect of Caregiver Education Level on Oral}

Health Behavior for Self and Child

Prior studies report that adult patient's education level indicated the strongest relation with health care behaviors of illness than other socioeconomic factors such as occupa- 
tion and income level: adults who are well educated perform more desirable health care behaviors such as routine check-up than those who are poorly educated [Liu et al., 2011; Winkleby et al., 1990]. Furthermore, a dental study indicated that adults with higher education were more likely to perform preventive oral health behaviors such as toothbrushing [Syrjala et al., 2002]. Our findings indicate significant influence of educational level on caregiver dental visits and routine dental care visit behavior, but not toothbrushing behavior. Our failure to find a relationship between education and tooth brushing behavior may have been influenced by an expectation of social norms, leading to social desirability bias. Interestingly, there was also no direct effect of caregiver education level on child's oral health behavior, but the association was indirect and mediated by caregiver oral health behavior. Our results are in conflict with other studies that have reported such a direct association [Camargo et al., 2012; Edelstein, 2002; Rajab et al., 2002; Sohn et al., 2007]. Enhancing caregiver's oral health behaviors may help improve children's oral health behavior, which in turn can reduce children's dental caries.

\section{The Direct Effect of Caregiver Education Level on the}

Children's Caries

We found a direct influence of caregiver education level on children's dental caries. This result indicates that other unobserved mediators not included in our model could influence the pathway between caregiver education level and children's decayed teeth. From a biological stand point, caregiver education level should not have a direct effect on children's dental caries. For example, biological mothers' education levels influence their own health behavior such as malnutrition or alcohol usage during pregnancy [Kalanda et al., 2006; Sant'Anna and Tosello, 2006], which can increase the likelihood of developmental enamel defects in their children, and subsequently dental caries [Targino et al., 2011]. On the other hand, microbiological variables such as early cariogenic bacteria colonization [Wan et al., 2003], and protective variables such as fluoride use [Feldens et al., 2010], lie in the pathway between caregiver education and children's caries. Similarly, other behavioral variables such as diet and drink consumption can also be potential mediators [De Coen et al., 2012; Vereecken et al., 2004]. Future research should include all behavioral, biologic, and preventive factors as mediators to identify the causal pathways between caregiver education and children's dental caries. Such a comprehensive model would be able to inform effective prevention strategies for young children's dental caries.

\section{Limitations of This Study}

There are limitations to our study. First, because we used a secondary dataset, the variables available were not sufficient to investigate all possible pathways. For example, we were not able to examine the biological pathway including variables such as the level of cariogenic organisms in the plaque or developmental defects. These variables may also influence caries status. Second, we recruited children and caregivers from one urban school district, and they may not represent other low-income African American families, which limit generalizability. Nevertheless, this study has a large sample size, which provides critical data on the effect of caregiver education levels on children's dental caries; this can help find efficient oral health promotion and prevention strategies. Additionally, it helped build relationships with this community and highlighted the importance of caries and dental care access. Third, caregiver education level and oral health behavior variables were collected by a self-reported-questionnaire. Thus, the responses might be influenced by recall and social desirability bias.

In conclusion, we found that in low-income African Americans, caregiver education level indirectly influenced their children's dental caries through the mediating influences of caregiver's and children's oral health behavior. Our results also indicate that improving caregiver oral health behavior might ameliorate the effects of low caregiver education and improve the children's behaviors and subsequent caries experience.

\section{Acknowledgment}

This study was supported by Grants HRSA/MCHB R40MC07838 and CTSC UL1 RR024989 from the Health Resources and Services Administration and the National Center for Research Resources respectively.

\section{The Role of the Authors}

Masahiro Heima: Generating this research project, analyzing data, and writing the manuscript.

Wonik Lee: Analyzing data, interpreting data, and writing the manuscript.

Peter Milgrom: Advising of the writing of the manuscript and revising the manuscript.

Suchitra Nelson: Advising of research project and revising the manuscript.

\section{Disclosure Statement}

All authors declare no conflicts of interest with respect to the authorship and publication of this article. 


\section{References}

al-Shalan TA, Erickson PR, Hardie NA: Primary incisor decay before age 4 as a risk factor for future dental caries. Pediatr Dent 1997;19:3741.

-Albert JM, Nelson S: Generalized causal mediation analysis. Biometrics 2011;67:1028-1038.

Bandura A: Principles of behavior modification. New York, Holt, Rinehart and Winston, Inc. 1969.

- Camargo MB, Barros AJ, Frazao P, Matijasevich A, Santos IS, Peres MA, Peres KG: Predictors of dental visits for routine check-ups and for the resolution of problems among preschool children. Rev Saude Publica 2012;46:87-97.

De Coen V, Vansteelandt S, Maes L, Huybrechts I, De Bourdeaudhuij I, Vereecken C: Parental socioeconomic status and soft drink consumption of the child. The mediating proportion of parenting practices. Appetite 2012;59:76-80.

$>$ Dulgergil CT, Colak H: Do the more caries in early primary dentition indicate the more caries in permanent dentition? Results of a 5-years follow-up study in rural-district. J Int Soc Prev Community Dent 2012;2:48-52.

Edelstein BL: Disparities in oral health and access to care: findings of national surveys. Ambul Pediatr 2002;2:141-147.

Feldens CA, Rosing CK, dos Santos BZ, Cordeiro MM: Pattern of fluoride-containing dentifrice use and associated factors in preschool children from Ijui, South Brazil. Oral Health Prev Dent 2010;8:277-285.

Grembowski D, Spiekerman C, Milgrom P: Linking mother and child access to dental care. $\mathrm{Pe}$ diatrics 2008;122:e805-e814.

Hooley M, Skouteris H, Boganin C, Satur J, Kilpatrick N: Parental influence and the development of dental caries in children aged 0-6 years: a systematic review of the literature. J Dent 2012;40:873-885.
Ismail AI, Lim S, Sohn W, Willem JM: Determinants of early childhood caries in low-income African American young children. Pediatr Dent 2008;30:289-296.

Kalanda BF, Verhoeff FH, Brabin BJ: Chronic malnutrition in pregnant adolescents in rural Malawi: an anthropometric study. Acta Obstet Gynecol Scand 2006;85:33-39.

Laaksonen M, Rahkonen O, Karvonen S, Lahelma E: Socioeconomic status and smoking: analysing inequalities with multiple indicators. Eur J Public Health 2005;15:262-269.

Lee W, Spiekerman C, Heima M, Eggertsson H, Ferretti G, Milgrom P, Nelson S: The effectiveness of Xylitol in a school-based clusterrandomized clinical trial. Caries Res 2014;49: 41-49.

Liu Q, Wang M, Guo J, Li J, Li C, Qian M: Effect of socioeconomic status on secondary prevention of stroke. Int J Qual Health Care 2011; 23:405-412.

Nelson S, Eggertsson H, Powell B, Mandelaris J, Ntragatakis M, Richardson T, Ferretti G: Dental examiners consistency in applying the ICDAS criteria for a caries prevention community trial. Community Dent Health 2011; 28:238-242.

Newton JT, Bower EJ: The social determinants of oral health: new approaches to conceptualizing and researching complex causal networks. Community Dent Oral Epidemiol 2005;33: 25-34.

Pitts N: 'ICDAS' - an international system for caries detection and assessment being developed to facilitate caries epidemiology, research and appropriate clinical management. Community Dent Health 2004;21:193-198.
Rajab LD, Petersen PE, Bakaeen G, Hamdan MA: Oral health behaviour of schoolchildren and parents in Jordan. Int J Paediatr Dent 2002;12: 168-176.

Sant'Anna LB, Tosello DO: Fetal alcohol syndrome and developing craniofacial and dental structures - a review. Orthod Craniofac Res 2006;9:172-185.

Sohn W, Ismail A, Amaya A, Lepkowski J: Determinants of dental care visits among low-income African-American children. J Am Dent Assoc 2007;138:309-318.

Sterne JA, White IR, Carlin JB, Spratt M, Royston P, Kenward MG, Wood AM, Carpenter JR: Multiple imputation for missing data in epidemiological and clinical research: potential and pitfalls. BMJ 2009;338:b2393.

Syrjala AM, Niskanen MC, Knuuttila ML: The theory of reasoned action in describing tooth brushing, dental caries and diabetes adherence among diabetic patients. J Clin Periodontol 2002;29:427-432.

Targino AG, Rosenblatt A, Oliveira AF, Chaves AM, Santos VE: The relationship of enamel defects and caries: a cohort study. Oral Dis 2011;17:420-426

Vereecken CA, Keukelier E, Maes L: Influence of mother's educational level on food parenting practices and food habits of young children. Appetite 2004;43:93-103.

Wan AK, Seow WK, Purdie DM, Bird PS, Walsh LJ, Tudehope DI: A longitudinal study of streptococcus mutans colonization in infants after tooth eruption. J Dent Res 2003;82:504508

Winkleby MA, Fortmann SP, Barrett DC: Social class disparities in risk factors for disease: eight-year prevalence patterns by level of education. Prev Med 1990;19:1-12. 